$I^{\circ}$ on the longitude

400 gon
100 gon
1 gon
1 gon
1 cgon
I mgon
I dagon
1 cgon on the latitude
I gon on the longitude

$=360^{\circ}$

$=90^{\circ}$

$=0.9^{\circ}$

$=$ 1 00 cgon

$=1$ centigon

$=1$ milligon

$=\mathrm{I}$ dekagon $\hat{=} \quad 4$ minutes of time

$=5400^{\prime}$

$=54^{\prime}$

$=1000 \mathrm{mgon}$

$=0.01$ gon $=0.54^{\prime}$

$=0.001$ gon $=0.054^{\prime}$

$=\quad \mathrm{r} \circ \mathrm{gon}$

人 $\quad \mathrm{r} \mathrm{km}$ (approx)

= 3.6 minutes of time

The abbreviation 'n.m. ', which stands for nautical mile, should not be confused with the designation ' $\mathrm{nm}$ ' that stands for nanometre $(0.000000001 \mathrm{~m})$.

The designation 'dagon' stands for dekagon ( $\mathrm{\circ} \circ \mathrm{gon}$ ) and it should not be confused with 'dgon' for decigon ( 0.1 gon). It can be used in two ways, namely (A) 23 dagon $=23^{\circ}$ gon and $(B) 23$ dagon $=225^{-235}$ gon.

The designation 'gon' is increasingly used in many languages, but the traditional words are as follows:

\begin{tabular}{lllll}
\hline & English & French & German \\
\hline${ }^{\circ}$ & degree & un degré & ein Grad \\
gon & grade & un grade & ein Gon \\
\hline
\end{tabular}

It is important to find out, at an early stage, which units and prefixes are to be preferred for different purposes in order to avoid a great variety followed by changes and confusions. The following units and examples of their uses are recommended.

\begin{tabular}{lllll}
\hline $\mathrm{km}$ & $\mathrm{km} / \mathrm{h}$ & \multicolumn{2}{c}{ gon } & \multicolumn{2}{c}{ cgon } \\
\hline length & speed & compass & angle & position \\
distance & current & variation & direction & latitude \\
range & tide & deviation & bearing & longitude \\
sight & ice drift & course & fairway & altitude . \\
visibility & wind speed & leeway & azimuth & sextant \\
\hline
\end{tabular}

KEY WORDS

1. Terminology 2. Display of information. 3. Units

\title{
Fixed and Floating Structures - Maritime Risk Assessment and Desiderata for Safe Navigation
}

\author{
R. St J. Fancourt
}

This paper is based on a contribution to Diagnostic Engineering, No. 55, by permission of the Institution of Diagnostic Engineers.

This paper considers an involuntary collision between a vessel of significant displacement and a captive major offshore installation where the energy at impact will be massive, 
deriving from the generated momentum in the form of kilotonnes of displacement times a factor for entrained water times velocity (knots), such that the result will be catastrophic in terms of cost, the environment or life. Involuntary is defined as other than deliberate or criminal action by those persons onboard the structure or those onboard the vessels in her offing.

An involuntary incident implies errors or failures or a combination of both such that an unintentional happening occurs. In essence, there can be but two principal parties: the striker and the stricken. Both are answerable for the fact that they were in that place, at the time, in that condition and for all steps leading thereto. Circumstances may be complicated by the presence and density of other marine traffic, by the presence of vessels whose task it is to service such installations, and by particular environmental factors.

In about 100 B.C. Polybius said: 'The surest and indeed only method of learning how to bear bravely the vicissitudes of fortune is to recall the calamities of others.' If we apply this dictum, we assiduously examine the scene, the steps and the options taken or not taken which, step by step, have led to, or are likely to lead to, the involuntary conclusion. This implies a systematic examination of the scene, and it identifies the methodology of assessment; that is, the identification of a series of risk elements whose individual resolution must stack up a particular way for there to be an incident. This precept explains why there are many more near-misses than incidents. In other words, a deteriorating situation can be improved or worsened by actions taken or not taken at any due time of decision. Time is ongoing and allows for late or early human realization. However, lapse of time implies changed circumstances which affect the correctness of any decision at the time of making it - the 'too little, too late' syndrome. The foregoing remarks are true for all human activities. In the maritime context we can catalogue the main headings of causative factors and relate them so that they represent a prognosis of calamity or a check-list for success. Ideogram $\mathrm{I}$ is offered as an overview of incident potential.

A deteriorating situation generated by:

(1) Actions of the striker

FaILURE: to have correct charts and notices

to plan and maintain a safe course in the prevailing circumstances

to detect deteriorating circumstances, e.g. environmental and traffic

to detect a hazard visually, by radar or other means

to appreciate and plan for equipment failures, e.g. radar, compass, steering gear, main engines and other

(2) Actions of the stricken

FaILURE: to identify adequate danger zone of own domain

to give adequate public notice of position and change of position when on semi-permanent location

to adequately notify when involuntarily out of station

to make provision for failure of surveillance radar beacons and communication channels with stand-by vessel and others

Ideogram I. Incident potential

As may be expected, a stricken installation's potential for influencing the risk of calamity comprises mostly open-loop influences. Compared with the other party, it has 
fewer time-related risk-element options. Nevertheless, all can be, and have been, main contributory factors, and an example could be a semi-permanent installation moving station, inadequately indicating the change and then, say, experiencing a shut-down. On the other hand, the striker is in the position of a closed-loop controller of events. The vessel may have the seeds of disaster sown in incorrect charts but she also has the opportunity yet to avoid calamity until the penultimate moment.

Prudent analysts eschew the easy option of merely identifying pilot error - human navigational error - when in fact erroneous judgements are contributed to by, say, failure of warning systems at a critical time or maladroit siting of controls. Such observations are of little comfort to a stricken installation, but they are nevertheless wholly relevant to safer and better-managed ships and a reduction of risk. Also, in considering the ongoing process of calamity-avoidance by all parties, it is to be noted that open-loop influences are ongoing in relation to the stream of risk (they are frequently less costly in implementation). In contrast, the closed-loop influences or controls in any system must be separately and individually exercised on each risk occasion within the ongoing stream of risk generation.

Within this scenario we may start with the premise that an operation or event is planned with an acceptable degree of risk. We can then analyse a series of happenings and assess their effect on the original level of risk as events unfold. The scene is depicted in Ideogram 2.

\begin{tabular}{lll}
\hline EVENT/FAILURE $\longrightarrow$ & Detected and heeded? \\
Moving ship & Stable \\
$\begin{array}{l}\text { Incorrect charts on board, erroneous passage plan } \\
\text { Other warning signs and signals, visual aspect, range } \\
\text { and bearing }\end{array}$ & Stable \\
$\begin{array}{l}\text { Course-keeping and positional errors } \\
\text { Other inputs }\end{array}$ & $\begin{array}{l}\text { Exacerbated } \\
\text { Erroneous definition of position } \\
\text { Power/lighting failure on installation } \\
\text { Congestion, other vessels confounding manoeuvres } \\
\text { Deteriorating weather conditions }\end{array}$ & $\begin{array}{l}\text { Exacerbated } \\
\text { Exacerbated }\end{array}$ \\
\hline
\end{tabular}

Ideogram 2. Vessel on passage in vicinity of offshore installations. Planned route with predetermined degree of risk

This method of examining the progression demonstrates the potential for a deteriorating situation and the need for alertness to perceive the untoward, the need to heed - in other words, the need to arrive at the correct judgement and action in the circumstances. It can be seen that a vessel can be wholly master of her destiny since she can correct for everything happening within her own systems. But where other inputs to the scene occur, all other factors being the same, an increased risk will occur and the vessel may or may not be able to cope. This is the stuff of near-misses and the reason for the need to plan for untoward contingencies.

In describing the scene in this way we introduce the human factor, the process of 
heeding, which can be described as receiving inputs of information, assessing the vital factors and arriving at the correct judgement in the circumstances. This definition is true of all endeavours undertaken by man, whatever the undertaking, whatever the environmental conditions. We are able to construct a causal-relationship diagram integrating man, machinery, environmental conditions and success or failure in the aim or object of the endeavour. Ideogram 3 is offered as an example of the interplay of these factors in the maritime scene. It may be used to analyse any untoward event.

\section{ENVIRONMENTAL CONDITIONS}

Daylight, twilight, darkness, fog, rain, snow, ice, storm, lightning, other, placid, tumultuous - severe ship motion

Consistent, sudden change

Shallow-water restraints, strong tidal/current effect

Proximity to land and other dangers, fixed and floating

\section{SHIP / MACHINERY / EQUIPMEN T}

Overall integrity affected by involuntary shift of weights due to poor stowage, ingress of water, accretion of ice, other

Quality, siting (usability) and maintenance of :

Radar, gyro, autopilot, sounder, satnav

Main engine and auxiliaries, deck, fire-

fighting, L.S.A.

Anchor and cables, steering gear

Hull and other openings

Other

\section{MAN (PERSON)}

Category

In charge, operative

Condition

Adequate training, adequate experience, external distraction, monotony, tiredness, illness, poor sight, stress, seasickness, drunkenness/other abuse Action/performance

Man can/may :

(mis)observe instruments

(mis)understand instructions

(mis)calculate position, etc.

select (un)wise or (im)proper manoeuvre (fail to) appreciate signs and signals, and critical situation, be neglectful and become torpid

SUCCESS OR CALAMITY

Collision, grounding, capsize, other

Ideogram 3. Causal relationship diagram

We now have a map of human endeavour which will enable various paths to be identified. For example, the planner is reminded to assess the risk of more or less severe environmental circumstances. He will be wise to have in mind the fact that no outfit of machinery and equipment is perfect all the time and of such a design that it cannot be misused. The awesome list of human fallibilities will remind him to consider crew fitness, training and experience in the particular circumstances. The path of events leading to a calamity (or near-miss) can be identified. In most cases it will be a complex chain of events linking, for example, darkness, storm, sudden change and shallow-water restraints, and where the person in charge is suffering from tiredness and an operative lacking adequate experience misreads an instrument and selects an unwise or improper manoeuvre. An alternative scenario might be failure to detect an instrument or equipment malfunction.

This introduces the distinction between mechanical failure and human (operational) failure. It is not a wholly satisfactory distinction since a mechanical failure implies an operational failure further back up the line of responsibility for maintenance and, indeed, 
a concurrent operational failure if there be no contingency for the unexpected; steering gears do jam hard over at the most inauspicious moments. However, staying with these two categories (mechanical failure and operational failure or error), simple examples might be something breaking or ceasing to function and somebody pulling the wrong lever or switch, it is worthy of note that a mechanical failure is immediately a new input on the operational scene and it will affect the operational decision-making process. A sudden failure is a stressful event and an operative may lack the experience necessary for the correct reaction. Also, those in operational control are subject to other stresses, like the deterioration of weather conditions or a near-miss with a seemingly rogue vessel, comparable to the 'mad drivers' that appear to be on the roads. For these reasons, crew training and simulated emergencies are vital in tuning and sustaining the correct human response under stress. Going to Emergency Stations as a routine on Thursday afternoon may not be sufficient.

It has to be acknowledged that calamities do occur, and will continue to occur, but it is important to strive to reduce them. When one has occurred there is the inevitable question - why did it occur in the manner it did? Very properly there are public inquiries so that lessons may be learned such that a similar calamity may never happen again. Another fruitful avenue of inquiry is the search for an answer to the question, Why did it occur when it did? 'When' implies time, the ongoing division of duration, and a lapse of time must mean the potential deterioration of both men and machinery. On the one hand, man needs sustenance. rest, activity, acknowledgement, training and experience to succeed. On the other, machinery needs sound design and monitoring of its condition for timely intervention in respect of its inevitable deterioration. Then linking the two, the interface must be ergonomically efficient. The lack of user-friendly controls spells disaster, and much marine equipment is poor in this regard. The scene can be viewed as in Fig. I.

In the maritime scene, as compared to aviation, less attention is paid to the general standard of engineering maintenance of all artefacts, and to the condition of persons onboard. One valid reason for this is the difference in lapse of time per identifiable task or operation. Aircraft can only stay in the air for a matter of hours, whereas ships steam for a matter of months with seemingly all systems unaffected by the passage of time.

Man is basically diurnal. He sleeps at night, he rises with or before (or after) the Sun, he does his work and retires or burns the candle at both ends. Particular arrangements are made to sustain particular duties and tasks such as night-duty, night-shifts and watches, whilst humdrum administration continues by day. It is of passing interest that night-shift production often exceeds that of day-shift - there are fewer distractions. However, such activities are for a finite length of time before the individual returns to normality and rotas are changed. In the aviation industry where day and night become merged, there is strict legislation as to time on duty and time for the body and mind to recover. In the maritime scene there is but one master and a finite number of crew who divide the day and night into watches as best they may. Ever since man first went avoyaging, the First Watch was not the first watch of the day, it was the first watch of the night; that is, all were generally available by day and the night was divided amongst those who watched. Nowadays, such distinctions are blurred. Nevertheless, the day frequently demands duties over and above the watch and the mariner must get his rest when he can. At times, typically at the beginning and end of a voyage and therefore continuously during short voyages, those on board are under severe pressure, sometimes more than is realized. This can be the reason for a higher proportion of marine accidents occurring during the hours of darkness, and hence discussion about the need for 


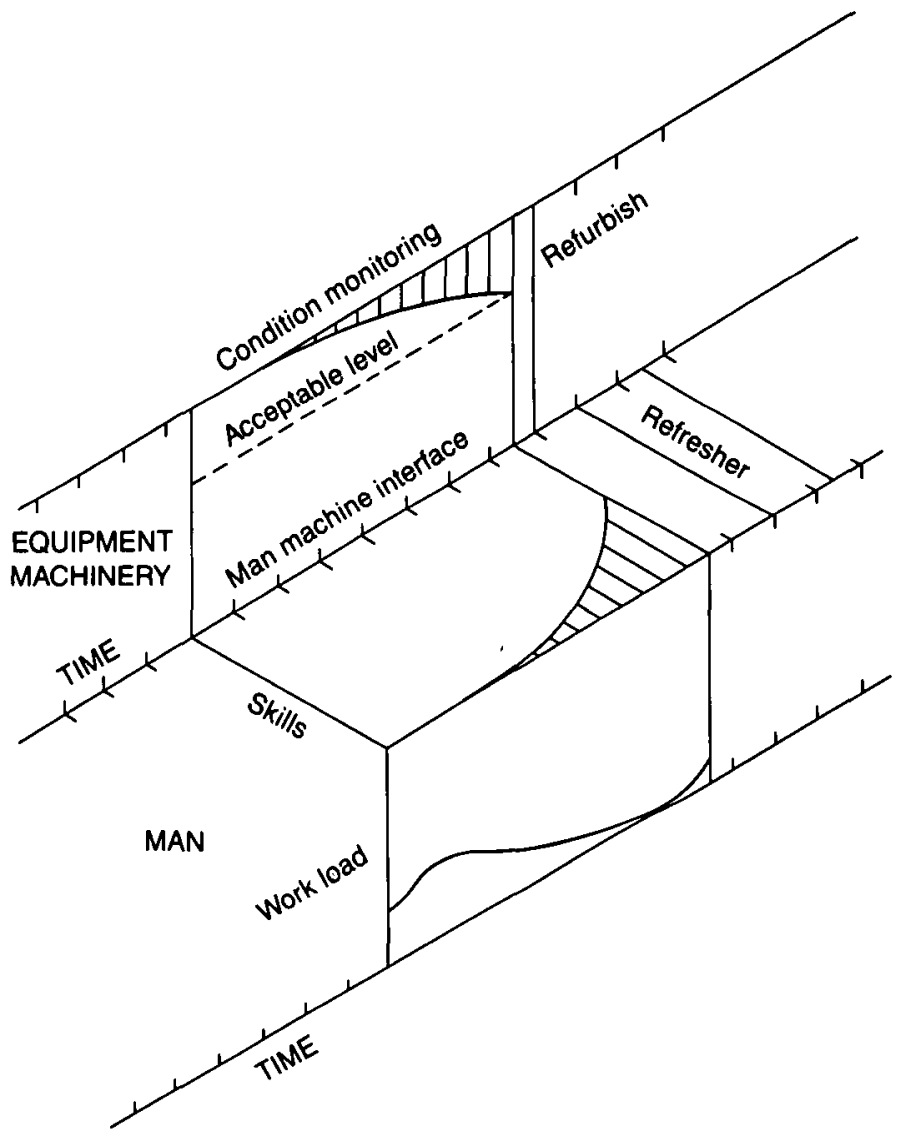

Fig. 1. The hysteresis of deterioration

legislation limiting the hours of duty in congested waters. However, ships will continue to traverse the trade routes of the world until day and night come to an end.

The total number of ships in the world is in the order of 60,000 . Approximately 40,000 may be voyaging at any one time and, of these, approximately 10,000 will be fishing vessels working in known defined areas. Therefore there will be a balance of approximately 30,000 ships voyaging in the trade routes of the world.

The annual average number of vessels experiencing a major incident is in the order of 1,000 marine casualties from all causes. Some of these occur when vessels are at anchor or in harbour and some where the vessels have limited geographical range (such as ferries, Mississippi paddle boats, fishing vessels, etc.) and we may therefore estimate, say, 200 incidents involving trading and similar vessels when proceeding on a voyage.

The overall annual incident rate of 60,000 ships experiencing 1,000 calamities, $1: 60$, and the rate for voyaging vessels, $1: 150$, are both cause for concern. However, these global figures may be looked at in terms of their effect at any one place. Those 200 ships due to be caught in the web of calamity will be voyaging in the trade routes of the world of some 3,750,000 square nautical miles. Within those routes the mean annual risk of a moving-ship incident at any one area of 1 square nautical mile will be $1: 18,750$. The odds will shorten in places of increased traffic density.

It has been estimated that there are 30,000 ships capable of unrestricted voyaging at 
any one time and that there will be 200 ship calamities in any year from amongst those ships. Let it be further assumed that those ships on the move are considered to be a risk to others for one hour when passing or otherwise in proximity. In those circumstances, the total yearly hours of risk will be $30,000 \times 365 \times 24$. If at any one location ships are known to pass at a rate of three per hour, the total annual hours exposed to risk will be $3 \times 365 \times 24$. Thus, that place bears $3 \div 30,000$ of the annual global risk and, with global incidents of 200 per annum, it bears an annual risk rate of $2 \times 10^{-2}$ (depicted in Fig. 2).

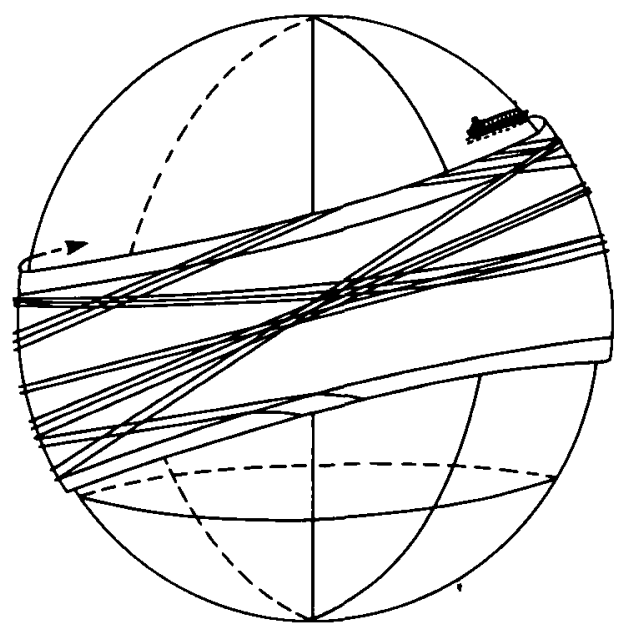

Fig. 2. The conveyor belt of commerce

These rounded values can be the subject of debate in presentation, meaning and assessment of the degree of risk, but however they are presented, there can be little room for complacency. Voyaging in the oceans of the world is a risky business (much more so in some places than others); consequently it is necessary to marshal a diagnostic prognosis of all risk-contributing elements.

There will be an increased risk of calamity where:

(i) Marine traffic is dense due to : deep-laden ships being restricted in movement, a confluence or crossing of traffic lanes, proximity of limiting hydrographic features, proximity of major ports and terminals, the presence of other activities, e.g. fishing, dredging, the presence of fixed and floating structures (the more so when their siting will cause an extension of a demanding or arduous passage in restricted sea room, where the structure is in an exposed flank position - last in the line - where it can act as an informal mark or beacon), and where product, maintenance and servicing traffic necessary for a group of installed structures compounds the existing traffic volume.

(ii) Inclement meteorological conditions occur - fog, poor visibility from any cause, storm, ice, etc.

(iii) During the onset of sudden change, during the hours of darkness and when untoward tidal/current influences occur.

This catalogue will assist in assessing the risk at any one place in relation to another, but to improve the overall risk (that is, to effect a reduction in the number of calamities) it is necessary to examine the steps necessary to improve the awareness, skills and effectiveness of those responsible for decision and action - those in the hot seat. Assessing awareness means examining all forms of communication and the correct input of information. This begs the question; are those responsible for timely decisions equipped with the wherewithal to gauge correctly the true scene? In yesteryear, the means were 
a chart (and notices), a compass, a log, a lead, sharp eyes and ears and an anchor. Nowadays, there is greater urgency, yet it is still vital to consider the tools-of-the-trade for the trade that the ship is in. There is a plethora of equipment to chose from, and there are regulatory minima which need constant review. Human skills and effectiveness are related to training, experience and condition at any one time, and the threat of fatigue is ever present. Deliberation and regulation of both the mechanical wherewithal and its optimum human use are therefore, perforce, a matter for international maritime organizations. Nevertheless, individuals and groups must contribute to those deliberations so that the closed-loop control of a moving object passing a stationary one is tuned as best it may be. Similarly, those responsible for maritime safety onboard an installation are contributors to the arena and to the decisions made therein. Their toolsof-trade are typically surveillance radar, communications with marine and air traffic, safety vessels and those with vicarious responsibility for the safety of the domain.

Should a careful prognosis of all factors indicate an unacceptable degree of risk, the solution can only be found by modifying the arena in some way. Options might include publicly declaring a larger domain, the installation of satellite low-risk equipment, an increase in the number of safety vessels stationed in the vicinity of a perceived threat and, perhaps in the last resort, equipped to effect physical intervention.

Lessons may be learned from other areas involving a high density of marine traffic. In the controlled circumstances of the major canals and waterways, few major calamities occur due to moving traffic, so it may be argued that it would be desirable to legislate for all passing vessels to report to an offshore guard cutter or other policing vessel, for short-lived permission to pass through a declared zone. An ultimate restriction would be to permit transit only by vessels proceeding in convoy. All such schemes have limited historical precedent and then usually in the context of hostile act rather than human waywardness, save for the example of the need to convoy ships in the frozen wastes of the north, where the environment imposes its will.

Having surveyed the overall maritime risk scene and the contributory factors therein, it is fitting to dwell on the task of the navigator, who, by definition, is responsible for safe and timely locomotion from one place to another, for, when that is consistently achieved, there can be no calamity.

Lecky in his Wrinkles in Practical Navigation stated that the navigator must first be experienced in the skills of seamanship. In those days, flying machines had not been invented and nowadays, an equally valid dictum would be that the pilot of an aircraft or a flying machine must first acquire the skills of airmanship to succeed in that environment. The first essential in respect of airmanship is the knowledge and skills necessary for sustaining the flying machine in the air, the chosen medium. The same is true for seamanship but, in the maritime scene, by the nature of the medium, the floating machine demands particular skills which are less continuously critical in maintaining equilibrium. Nevertheless, the effects of the environment on the medium are frequently critical.

Man has been devising floating machines since time began, but those designs have not been without shortcomings in relation to both the task and the environment. With the passage of time, requirements and designs have become more ambitious and they have sought to move greater payloads, faster and at less cost. These are worthy goals but, in so striving, an insidious change has taken place. Captain Cook, perhaps the most able navigator of all time, had the misfortune to strand his ship on a coral reef but, within that ship and of the persons onboard, he had the skills and the wherewithal to effect repairs such that the voyage could be satisfactorily completed. Great skills of seamanship were exercised and the only hurt to the environment was some damaged coral. Such is 


\section{SHIP'S DOMAIN - Intended ahead motion}

Total effective volume varies as:

Displacement $\times$ potential speed ${ }^{2} \times$ site factors,

e.g. depth of water under keel, reaction of obstruction, reaction due to shoaling etc.

\section{Impinging Forces:}

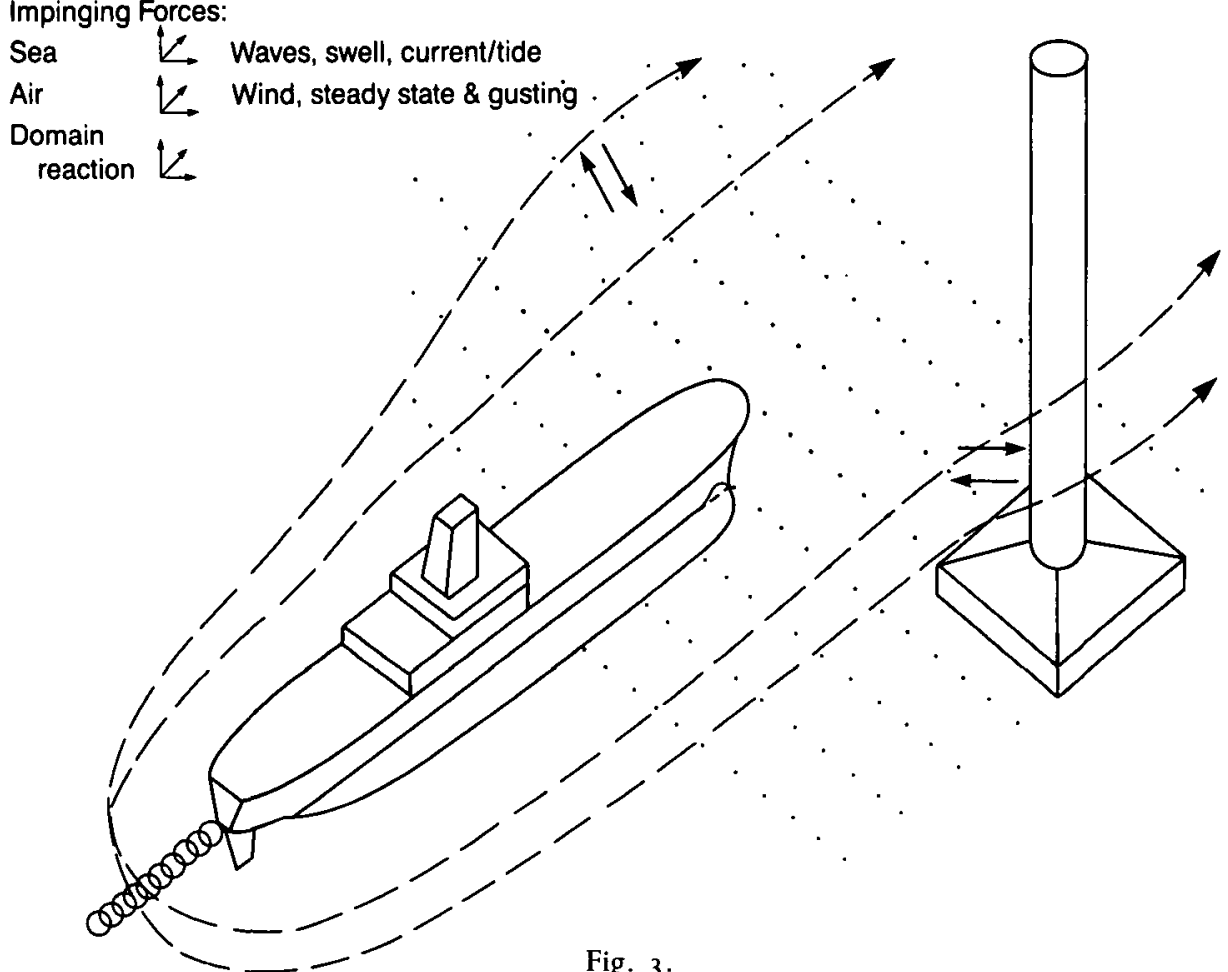

Fig. 3 .

not the case when a crude-oil or large carrier is stranded, since the man/ship system is not equipped to effect self-help. The seaman navigator is restricted in his ability to exercise seamanship by the very nature of current designs. Is this trend desirable and should it be addressed?

Impact between one ship and another or a fixed structure must involve velocity; if the navigator had sensors to detect impending impact and the wherewithal to stop his ship, involuntary collision would be obviated, subject to adequate and proper human control and the degree of risk that this entails. Wholly automated ships might be seen to be a solution but, in fact, the human imput is not removed: it is merely transferred to another place; that is, ashore, with the consequent risks of making those arrangements which, in total, may yield an adroit solution, like the routines involved in manoeuvring laden dumb lighters from shore to shore. The essence of the matter remains the safe and timely locomotion of that which is to be moved - the task of the seaman navigator who will need the best possible tools for the trade that he is exercising. How best to satisfy that need? Like a prudent navigator, we can take stock of the situation; we are in the business of safe and timely locomotion. At this juncture it may be said that the wherewithal and awareness of the locus is well developed, but that for controlled motion is less so. The factors highlighted in this paper suggest that the means and the awareness of the need for redirecting or stopping motion are matters which should be urgently addressed. 
Ultimately seamanship, like airmanship, is a matter of maintaining the ability to remain in the medium; that is, the ability to remain safely afloat. In addressing this perpetual requirement the seaman navigator has constantly to satisfy himself that in proceeding he is not standing into danger. Many dangers are locus-related and publicised, so that the modern voyager does not sally forth into the great unknown, as was often the case in the time of Captain Cook. Yet the number of maritime calamities remains constant, despite the continuing improvement of navigational aids.

Danger, hazard and peril are those things which frustrate the safe and timely conclusion of a voyage. They encompass, for example, volcanic islands rising out of the sea or, more usually, storms and other inclement weather which cause cargo to shift or cause dynamic failure and the ingress of water or, more usually still, the waywardness of other navigators. Of these categories, the first may be deemed an act of God in response to which man must act as best he can. The second calls for improvements in design, the better to cope with the onslaught of the elements. All call for prescience and seamanship, the better to cope with whatever situation arises.

The Rules of the Road provide statutory guidelines for both awareness and action but, in the last resort, the navigator must get and keep his ship out of harm's way as best he may. The essence of the matter is controlled motion, in the environment pertaining and in the domain of the ship. Particular force vectors have to be resolved or controlled if the ship and the persons onboard are not to become prisoners of circumstance, such as collision or being engulfed.

A moving floating machine, her entrained water and the water in her future path comprise the domain over which control is vital, and it follows that success must lie in the prescient control of the ship, which can only be achieved by knowledge of the particular characteristics of the particular ship, in the particular circumstances. Such is the essence of good seamanship and the foundation of safe navigation.

KEY WORDS

1. Risk analysis 2. Collisions

\section{'Trans-oceanic Passages by Rhumbline Sailing'}

\section{Professor Han-Fei Lu, Hsin-Hsiung Fang and Chung-Hsing Chiang \\ (National Taiwan Ocean University, Institute of Marine Technology)}

I. INTRODUCTION. In the paper 'Trans-oceanic passages by rhumbline sailing' contributed by Captain Ivica Tijardović, ${ }^{1}$ the following formulae were used to find the rhumbline course and derive the minimum distance by a differential method:

$$
\begin{gathered}
D=\delta \phi \sec \theta+(\delta \lambda-M \tan \theta) \cos \phi_{2}, \\
\sin \theta=M \cos \phi_{2} / \delta \phi .
\end{gathered}
$$

The terms in these equations are as defined in Captain Tijardović's Fig. I. The equations offer the mariner a simple and rational method of saving distance as compared with a direct rhumbline track between two positions, but some questions arise which are worthy of discussion. 